\title{
STUDY OF CRYPTOSPORIDIOSIS IN CHILDREN WITH GASTROENTERITIS IN AND AROUND BIDAR
}

\author{
Pramod S. Manthalkar1, Parmeshwarappa K. Dhannurkar², Chandrakant Chillarge ${ }^{3}$
}

1Assistant Professor, Department of Microbiology, Bidar Institute of Medical Sciences, Bidar. 2 Assistant Professor, Department of Microbiology, Bidar Institute of Medical Sciences, Bidar. 3Professor and HOD, Department of Microbiology, Bidar Institute of Medical Sciences, Bidar.

\section{ABSTRACT}

\section{BACKGROUND}

The aim is to study the prevalence of Cryptosporidium parvum in children suffering from gastroenteritis in and around Bidar.

\section{MATERIALS AND METHODS}

Total 200 samples were collected from the patients suffering from acute gastroenteritis. These samples were subjected for different staining and concentration methods for identification of Cryptosporidium parvum oocyst.

\section{RESULTS}

Out of 200 samples, 27 stool samples were positive for Oocyst of Cryptosporidium parvum, 22 for cyst and trophozoites of Giardia lamblia, 03 for cysts of E. histolytica, 06 for EPEC. Modified ZN stain was found to be the best to identify the Oocyst of Cryptosporidium parvum and Sheather's sugar flotation was found to be good.

\section{CONCLUSION}

Constant surveillance of stool sample is a must for timely formulation of environmental hygiene.

\section{KEYWORDS}

Cryptosporidium, Gastroenteritis, Modified ZN, Sheather's Sugar Flotation.

HOW TO CITE THIS ARTICLE: Manthalkar PS, Dhannurkar PK, Chillarge C. Study of cryptosporidiosis in children with gastroenteritis in and around Bidar. J. Evolution Med. Dent. Sci. 2017;6(28):2325-2328, DOI: 10.14260/Jemds/2017/500

\section{BACKGROUND}

Diarrhoeal diseases are the commonest infection in infants and children, which may be due to bacteria, viruses, parasites and fungi, etc. with the emergence of HIV and other immunocompromised diseases, parasites like cryptosporidium, isospora, microsporidia, etc. are responsible for causing diarrhoea.1,2 Organisms which were never known to cause disease in man are identified as responsible for causing severe infections in childhood. This may be due to better availability of the diagnostic facilities, increase in the better medical facilities, extensive use of antibiotics, corticosteroids and other drugs. ${ }^{3}$ Environmental factors play an important role in the causation of the diarrhoea through drinking water, food, sanitation and association with house hold animals, etc.3,4,

Childhood diarrhoea in developed countries is due to viruses and newer pathogens, due to their well-planned sanitation, vaccination. ${ }^{5}$ In India because vast population is uneducated, living in rural area, due to poorly planned sanitation facilities along with very high population growth, infectious diseases especially diarrhoea are responsible for causing high mortality and morbidity. ${ }^{6}$

Financial or Other, Competing Interest: None.

Submission 27-02-2017, Peer Review 24-03-2017,

Acceptance 30-03-2017, Published 06-04-2017.

Corresponding Author:

Pramod S. Manthalkar,

Assistant Professor,

Department of Microbiology,

Bidar Institute of Medical Sciences,

Bidar-585401, Karnataka.

E-mail: pramodmanthalkar@gmail.com

DOI: $10.14260 /$ jemds $/ 2017 / 500$
Enteric protozoan parasitic infection has become an important cause of morbidity in children and adults, not only in developing but also in developed countries. The major parasites implicated are Giardia lamblia and the emerging spore forming protozoa viz Cryptosporidium parvum and C. cayetanensis. ${ }^{6}$ C. parvum appears to have become a threat to public health as it is ubiquitous, highly resistant to disinfectants and to date no effective therapy is available. First reported by Tyzzer in 1907 and well known to veterinarians since 1953, Cryptosporidium has gained recognition as human pathogen since $1976 . .^{7}$ The AIDS pandemic has brought Cryptosporidium to the forefront as an important cause of life threatening infection in immunocompromised persons. In the developing world, the association of cryptosporidium with both acute and persistent diarrhoea among immunocompetent children has been striking (26.9\%). ${ }^{8}$

Cryptosporidium is a coccidian parasite which resides in the microvillus of the lumen and multiply in the microvillus producing Oocyst, leading to malfunction of the lumen. The major symptoms related to cryptosporidiosis are similar to that of the Cholera and other coccidian infections. That is why we have chosen this study.

Bidar being a small town in north Karnataka, majority of the people do farming and are exposed to animals, also as the boundaries are connected to Maharashtra and Andhra majority of the people migrate, there is no closed drainage system and people consume well water. Hence, we planned to identify the aetiological agent of diarrhoea in children and for demonstration of Cryptosporidium, and we planned to evaluate different demonstration techniques. 


\section{MATERIALS AND METHODS Sample Collection}

Detailed history was obtained from the paediatric department of the patients suffering from diarrhoea, also HIV status was obtained. A total of 200 patients were included in the study, out of 200 patients we were unable to get the detailed history of HIV in 5 patients. Rest all were HIV negative.

200 stool samples from paediatric patients suffering from diarrhoea were collected from Regional Diagnostic Laboratory Microbiology section, in a sterile wide mouth container.

Macroscopic examination of all the stool sample was recorded in the laboratory case sheet stating the colour, consistency, odour, presence of mucus, presence of blood, any segments of the parasites in relation to Helminths' infection.

Routine microscopy of stool sample including hanging drop preparation was done to rule out the possibility of Vibrio cholera infection and also to observe the motility of the trophozoite of the parasites.

All the stool samples were subjected for wet mount preparation, staining and concentration techniques. Microscopy of the stool sample was done, by subjecting the stool sample for saline and iodine mount preparation, for identification of trophozoite forms, eggs and oocyst and other infective agents. One drop of saline was taken on a new grease free slide and a loopful of stool sample was added to it and mixed well and coverslip was applied on it and observed under low power and high power objective. For iodine mount one drop of Lugol's iodine was taken on slide and a loop full of stool sample was added to it mixed well and observed under low and high power objective.

\section{Staining}

Smear was prepared for the portion of the stool sample and was air dried and fixed with acetone and stained with Modified acid fast staining (Cold method). The prepared smear was first flooded with dilute carbol-fuchsin and kept for $15 \mathrm{~min}$. and then decolourised with acid alcohol (1\%) and then slide was washed with buffer solution and then methylene blue was added to the smear and kept for $5 \mathrm{~min}$. and then buffer wash was given and air dried and observed under oil immersion objective. 2,5

Other stains that were simultaneously done by preparing the smear from the same sample and were subjected for Gram stain, Kinyoun's acid fast (Hot stain), Giemsa stain, PAS and Trichrome stain. 9,10,11,12,13,14

Stool concentration techniques: All the stool samples were subjected for concentration techniques, 1 gram of portion of stool sample was added to Sheather's sugar solution and mixed well and was centrifuged and then allowed to settle. After $10 \mathrm{~min}$., tube was filled with the sugar solution, then cover slip was placed on the mouth of the test tube and this coverslip was kept on the slide and observed under low power and high power for demonstration of Oocyst of cryptosporidium which floats on sugar solution. ${ }^{14,15}$.

Also, the same samples were subjected for formal ether concentration technique, for demonstration of oocyst of Cryptosporidium parvum. In this one gram of stool sample was taken and added to $10 \%$ formalin solution and mixed well and centrifuged, then the stool sample was removed and was kept in test tube rack to settle and then ether was added to the formalin mixed stool sample after $15 \mathrm{~min}$. With the help of pasture pipette the supernatant was taken and one drop was added on glass slide for demonstration of Oocyst, supernatant then was separated in a new test tube and sediment was examined by taking one loopful of stool sample from the bottom of the tube.

\section{RESULTS}

Total 200 samples were collected for the demonstration of various parasitic infections in children form paediatric ward and OPD. Out of 200 patients 30 patients were below 1 year, 107 were between 1 to 5 years and 63 were above 5 years of age. Of the 200 samples, 27 (13.5\%) stool samples were positive for Oocyst of cryptosporidium parvum, trophozoites and cyst of Giardia lamblia was seen in $22(11 \%)$ cases, Entamoeba histolytica in $03(1.5 \%)$ cases and also could isolate Enteropathogenic E. coli (EPEC) in 06 (3.0\%) cases of diarrhoea among children. In two cases, there was coinfection of E. coli and Giardia lamblia. Candida albicans was isolated from two stool samples.

Oocyst of Cryptosporidium was demonstrated in 27 patients, stool of the patients below one year were negative for Oocyst of cryptosporidium. Cryptosporidium was demonstrated in the age group between 1 to 5 years and above 5 years. (Table 1 ).

Out of 27 samples positive for Oocyst of Cryptosporidium parvum, modified acid fast stain gave $100 \%$ results while Giemsa, Iodine, PAS, Trichrome stain gave 11 (40\%), 16 (59.25\%), 19 (70.37\%) \& 22 (81.48\%) positivity respectively. Sheather's sugar flotation revealed $100 \%$ results, while formal ether and formalin sedimentation were 52\% and $13 \%$ positive respectively.

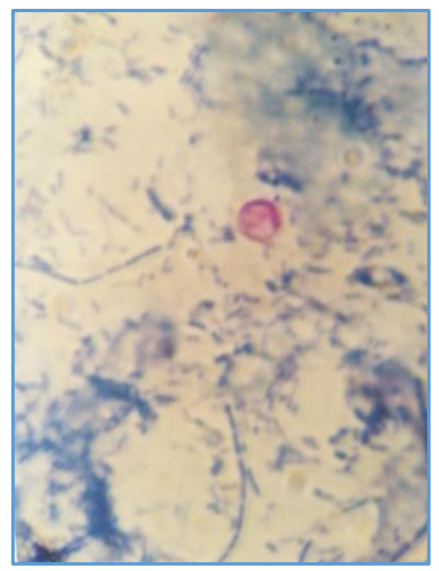

Figure 1. Oocyst of Cryptosporidium Parvum (Modified Acid Fast Staining)

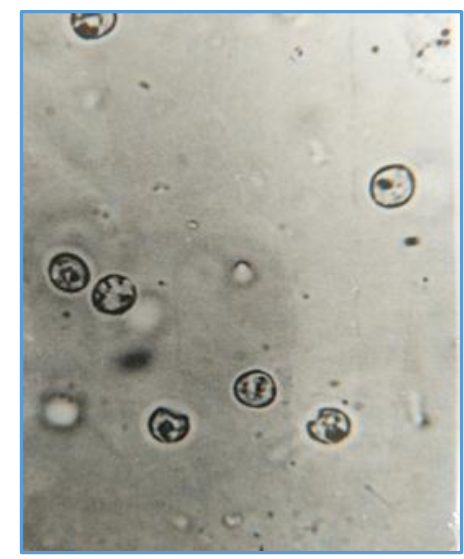

Fig 2. Oocyst of Cryptosporidium Parvum in Wet Mount (Sheather's Sugar Flotation Concentration) 


\begin{tabular}{|c|c|c|c|c|}
\hline \multirow{2}{*}{ Organism Name } & \multicolumn{3}{|c|}{ Age Group in Years } & \multirow{2}{*}{ Total } \\
\hline & $<1$ Year & 1 to 5 Year & $>5$ Year & \\
\hline Cryptosporidium & 00 & $11(42.3 \%)$ & $16(61.5 \%)$ & 27 \\
\hline Giardia lamblia & 00 & $03(13.6 \%)$ & $19(83.3 \%)$ & 22 \\
\hline E. histolytica & 00 & 00 & $03(1.5 \%)$ & 03 \\
\hline EPEC & 00 & $06(100 \%)$ & 00 & 06 \\
\hline Candida albicans & $02(100 \%)$ & 00 & 00 & 02 \\
\hline
\end{tabular}

\section{DISCUSSION}

Majority of the school children in India and other developing countries harbour one or more intestinal parasites, which are responsible for causing diarrhoea. Along with established intestinal parasites newer parasites are reported as emerging parasites, one such recently described parasite is Cryptosporidium parvum, as awareness increased and laboratory facilities were available, reports of cryptosporidiosis in immunocompetent individuals also emerged. Prevalence of human cryptosporidiosis varies widely in different part of the world. Guerrant RL reported cryptosporidium infection in $2.1 \%$ and $6.1 \%$ of immunocompetent individuals suffering from diarrhoea in developed and developing countries respectively. In our study, the analysis of data shows that causative relationship of cryptosporidium in children with diarrhoea compared to healthy controls to be statistically significant $(\mathrm{P}<0.001)$. In each geographical area, prevalence of the parasite reported varies widely such as Sethi $S$ et al found Cryptosporidial oocysts in $8.4 \%$ children suffering from diarrhoea and $5.9 \%$ in control group. Reports from South India has shown that $13.1 \%$ and $9.8 \%$ of children with diarrhoea and healthy control excreted the Oocysts. In West Bengal and Mumbai, the parasite was detected in $4.45 \%$ and $5.5 \%$ of children with diarrhoea. Study from Loni Maharashtra also shows lower prevalence in cases of diarrhoea. ${ }^{16,17}$

In our study, only HIV status was assessed as a predisposing factor in 40 children with diarrhoea and 04 controls, who showed the presence of cryptosporidium in their faecal samples but other conditions giving rise to immunocompromisation and immunocompetent individuals are extensively studied. Our findings of more severe clinical manifestations, more common in younger age group are consistent with review report by Fayer and Ungar and Malebrance $\mathrm{R}$ et al. Isaacs et al have also reported similar findings. We did not come across any child developing metabolic disturbance, DIC, toxic megacolon, post enteritis syndrome, etc.

In young children, breastfeeding has been reported to give immunity against the infection of this parasite. Reports from Costa Rica and Brazil show absence of Cryptosporidial infection in breastfed children. We also noted that parasite was less prevalent in breastfed children. Even Malla et al have reported similar observations but Mathan et al did not find any change in the prevalence of breastfed and TOP fed children.

Even though, we did perform Sheathe's sugar flotation technique for concentration of the parasite, it was observed that Cryptosporidial oocysts were present in plenty when they are aetiological agents. So concentration method is more useful for detection of carriers.
Cryptosporidium diarrhoea in children can lead to malnutrition, failure to thrive as long-term complications. ${ }^{18}$ So it is necessary to study its prevalence in each geographical area and examine the diarrhoeal stool for the parasite and also do the followup studies in these children.

\section{CONCLUSION}

Constant vigilance for newer parasites is a must for proper control of the infection in the community. Also different methods should be adopted for the demonstration of parasite for maximum output of the prevalence.

\section{REFERENCES}

[1] Kumar P, Vats O, Kumar D, et al. Coccidian intestinal parasites among immunocompetent children presenting with diarrhea: are we missing them? Tropical Parasitology 2017;7(1):37-40.

[2] Vanathy K, Parija SC, Mandal J, et al. Detection of Cryptosporidium in stool samples of immunocompromised patients. Tropical Parasitology 2017;7(1):41-6.

[3] Murugesan M, Ganesan SK, Sitara SR. Cryptosporidiosis in children in the Indian subcontinent. Tropical Parasitology 2017;7(1):18-28.

[4] Shrivasta AK, Kumar S, Smith WA, et al. Revisiting the global problem of cryptosporidiosis and recommendations. Tropical Parasitology 2014;8(11): DC07-DC08.

[5] Santosh KA. Microbiological stool examination: Overview. JCDR 2012;6(3):503-9.

[6] Quinn TC, Benden BS. New development in infectious diarrhoea. Disease a- month (XXXII). 1986:4.

[7] Lamber HP. The pathogenesis of diarrhea of bacterial origin. Recent Adv In Infection 1979;(1):109-20.

[8] Fayer R, Ungar BL. Cryptosporidium species and cryptosporidiosis. Microbiol Rev 1986;50(4):58-83.

[9] Levin ND. Taxonomy and review of the coccidian genus Cryptosporidium (Protozoan, Apicomplexa). J Protozool 1984;31(1):94-8.

[10] Upton SJ, Current WL. The species of Cryptosporidium (Apicomplexa: Cryptopordiidae) infecting mammals. J Parasitol 1985;71(5):625-9.

[11] Casemore DP. Timing of symptoms and oocysts excretion in human Cryptosporidiosis. New England Journal of Medicine 1987;317(3):168-9.

[12] Uppal B, Natarajan R. Detection of Cryptosporidium oocysts in acute diarrhoea stools. Indian Paediatric 1991;28(8):917-20.

[13] Anantha SM, Sanath S, Thyagarajan PS. Comparison of staining techniques employed in the detection of cryptosporidium oocyst in stool sample. Ind J Med Microbiol 1994;12(4):297-9. 
[14] Zierdt WS. Concentration and identification of cryptosporidium species by use of a parasite concentrator. J Clin Microbiol 1984;20(5):860-1.

[15] Ignatius R, Lehmann M, Miksits K, et al. A new acid-fast trichrome stain for simultaneous detection of Cryptosporidium parvum and microsporidial species in stool specimens. J of Clin Microbiol 1997;35(2): 446-9.
[16] Saraswathi K, Pandit DV, Deodhar LP, et al. Prevalence of Cryptosporidia in patients with diarrhoea in Bombay. Indian J Med Res 1988;87:221-4.

[17] Sethi S, Sehgal R, Malla N, et al. Cryptosporidiosis in a tertiary care hospital. National Med J India 1999;12(5):207-9.

[18] Guerrant RL. Cryptosporidiosis: an emerging, highly infectious threat. Emerg Inf Dis 1997;3(1):51-7. 